\title{
Controlling the Spontaneous Emission Rate of Quantum Wells in Rolled-Up Hyperbolic Metamaterials
}

\author{
K. Marvin Schulz, ${ }^{1,2, *}$ Hoan Vu, ${ }^{1,3, \dagger}$ Stephan Schwaiger, ${ }^{1}$ Andreas Rottler, ${ }^{1}$ Tobias Korn, ${ }^{4}$ David Sonnenberg, ${ }^{1}$ \\ Tobias Kipp, ${ }^{3}$ and Stefan Mendach ${ }^{1}$ \\ ${ }^{1}$ Institut für Nanostruktur und Festkörperphysik, Universität Hamburg, Jungiusstr. 11, D-20355 Hamburg, Germany \\ ${ }^{2}$ Institut für Optische und Elektronische Materialien, Technische Universität Hamburg-Harburg, D-21073 Hamburg, Germany \\ ${ }^{3}$ Institut für Physikalische Chemie, Universität Hamburg, Grindelallee 117, D-20146 Hamburg, Germany \\ ${ }^{4}$ Institut für Experimentelle und Angewandte Physik, Universität Regensburg, D-93040 Regensburg, Germany
}

(Received 29 February 2016; published 17 August 2016)

\begin{abstract}
We experimentally demonstrate the enhancement of the spontaneous emission rate of GaAs quantum wells embedded in rolled-up metamaterials. We fabricate microtubes whose walls consist of alternating $\mathrm{Ag}$ and $(\mathrm{In})(\mathrm{Al}) \mathrm{GaAs}$ layers with incorporated active GaAs quantum-well structures. By variation of the layer thickness ratio of the $\mathrm{Ag}$ and $(\mathrm{In})(\mathrm{Al}) \mathrm{GaAs}$ layers we control the effective permittivity tensor of the metamaterial according to an effective medium approach. Thereby, we can design samples with elliptic or hyperbolic dispersion. Time-resolved low temperature photoluminescence spectroscopy supported by finite-difference time-domain simulations reveal a decrease of the quantum well's spontaneous emission lifetime in our metamaterials as a signature of the crossover from elliptic to hyperbolic dispersion.
\end{abstract}

DOI: 10.1103/PhysRevLett.117.085503

Metamaterials are artificial compound materials with subwavelength inclusions which allow us to create optical properties on demand. The attainable optical properties in metamaterials are beyond that of ordinary materials found in nature and give rise to new optical phenomena including negative refraction of light [1-4], subwavelength imaging $[5,6]$, or invisible cloaking $[7,8]$. Besides that, metamaterials offer the unique opportunity to study quantum emitters in tailorable optical environments. It has been experimentally demonstrated that so-called hyperbolic metamaterials enhance the spontaneous emission rate of quantum emitters such as colloidal quantum dots and dye molecules when placed in close proximity to the material $[9,10]$ and also when positioned inside the material, which has been accomplished by infiltration into nanopatterned slots and a spin coating technique [11-13]. Likewise, hyperbolic metamaterials are capable of amplifying the surfaceenhanced Raman scattering of dye molecules when placed on top of the metamaterial [14]. The enhancement arises as a consequence of the unique dispersion relation of these materials [15-17]. In fact, the isofrequency surface in such materials is of hyperboloidal shape. This is in contrast to the majority of conventional materials, where the isofrequency surface forms an ellipsoid or sphere [18]. It is for this reason that hyperbolic metamaterials support an increased number of propagating light modes often associated with a singularity of the local photonic density of states (PDOS) [15-17]. An emitter in the vicinity or inside the hyperbolic metamaterial can couple to the hyperbolic modes, which can significantly enhance its radiative decay rate.

Here, we investigate the spontaneous emission lifetime of semiconductor quantum-well $(\mathrm{QW})$ heterostructures that are directly integrated inside rolled-up microtubes (RMTs) with hyperbolic dispersion. RMTs can be fabricated by self-rolling of strained semiconductor layer systems [19,20] and have been investigated as ring resonators that support azimuthal optical [21] and spin wave resonances [22] as well as plasmonic hybrid whispering gallery modes [23] that can be supported if a metal layer is introduced to the system and have been explored for sensing applications [24,25]. In our study, we use the rolled-up fabrication technique to realize hyperbolic metamaterials with integrated semiconductor quantum structures which enables us to study the spontaneous emission of quantum structures in a metamaterial with controlled optical parameters. In the rolling-up process, multiple functional layers are closely stacked on top of each other, forming a three-dimensional radial metamaterial that operates at optical frequencies. Rolling up metalsemiconductor systems is a unique and elegant route to incorporate optically active quantum-well structures directly inside a multilayer system and results in a high-quality active metamaterial composed of identical functional layers. Within this approach, the variable metal and semiconductor layer thicknesses allow us to control the effective permittivity tensor of the metamaterial. Thereby, the topology of the isofrequency surface can be changed from a closed ellipsoidal isofrequency surface to an open hyperboloidal surface corresponding to a hyperbolic metamaterial. We show by means of time-resolved low temperature photoluminescence (PL) spectroscopy supported by electromagnetic simulations that the spontaneous emission rate of the embedded GaAs quantum wells is enhanced by a factor of 2 as a signature of the topological transition to a hyperbolic medium.

RMTs are fabricated by using the self-rolling mechanism of strained semiconductor layers $[19,20]$. The utilized multilayer structure is shown in Fig. 1(b). In detail, on 


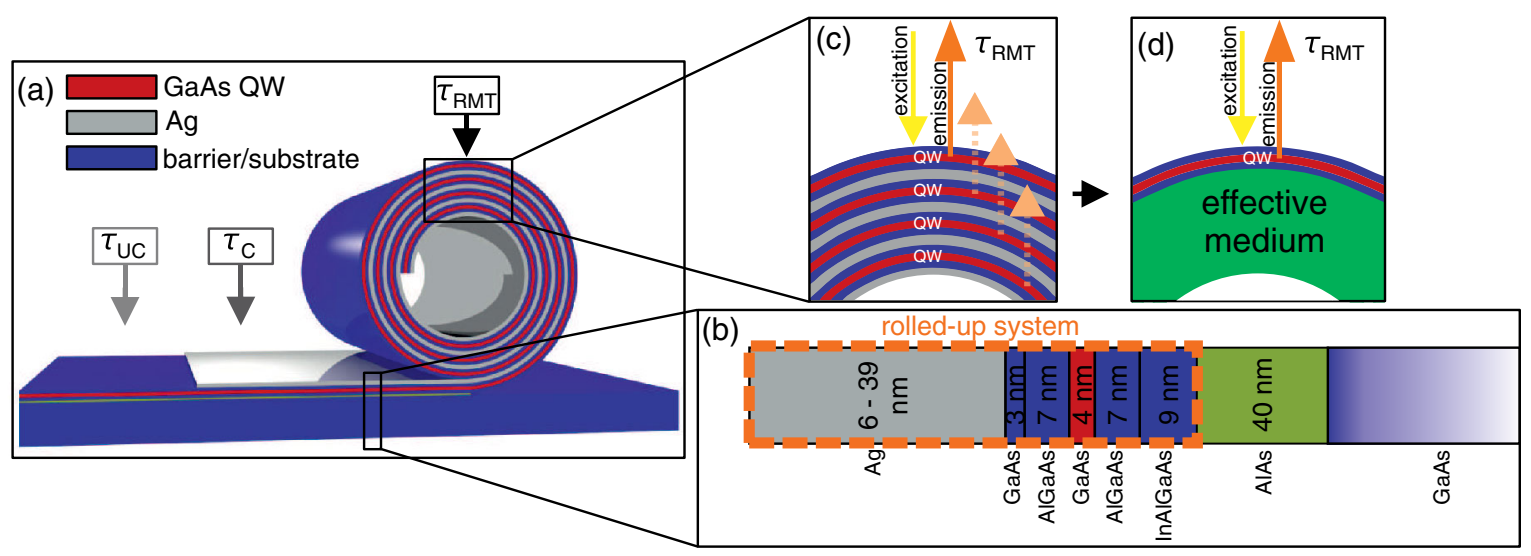

FIG. 1. (a) Sketch of the sample geometry showing a microtube (diameter $d \sim 5 \mu \mathrm{m}$ ) that was rolled up from the metal coated substrate. Four different samples were prepared each of which with a different Ag layer thickness ranging between 6 and $39 \mathrm{~nm}$. (b) Detailed multilayer structure with integrated QW structures from which microtubes were rolled up by self-rolling of strained layers. Layer composition: GaAs substrate layer, AlAs sacrificial layer (40 nm), InGaAs strained layer (9 nm), AlGaAs/GaAs/AlGaAs QW (7/4/7 nm), GaAs coating layer (3 nm), and Ag layer (6-39 nm). (c) Magnified wall of the microtube representing a three-dimensional radial metamaterial consisting of alternating $\mathrm{Ag}$ and GaAs QW layers. QWs in the tube wall emit radiation after excitation with the pump laser. Emission of lower lying QW layers is attenuated due to absorption in the tube wall. (d) Model of the microtube wall showing a QW layer emitting radiation on top of an effective medium.

top of a GaAs substrate an AlAs sacrificial layer (40 nm), an InGaAs strained layer $(9 \mathrm{~nm})$, an $\mathrm{AlGaAs} / \mathrm{GaAs} /$ AlGaAs QW (7, 4, and $7 \mathrm{~nm}$ ), and a GaAs coating layer $(3 \mathrm{~nm})$ are subsequently grown by molecular beam epitaxy (MBE). On top of this $30 \mathrm{~nm}$ thick semiconductor heterostructure, Ag layers of variable thickness $(6-39 \mathrm{~nm})$ are deposited by thermal evaporation that are partially coating the substrate. The rolling-up process is induced by selectively removing the AlAs sacrificial layer with hydrofluoric acid. This exposes the InGaAs strained layer that bends up while minimizing its strain energy leading to the formation of a microtube with an adjustable number of rotations. From the same MBE grown wafer we fabricate four different samples with $\mathrm{Ag}$ layers of thicknesses $d_{\mathrm{Ag}}=6 \mathrm{~nm}$, $d_{\mathrm{Ag}}=17 \mathrm{~nm}, d_{\mathrm{Ag}}=22 \mathrm{~nm}$, and $d_{\mathrm{Ag}}=39 \mathrm{~nm}$. These samples are denoted as $S_{6}, S_{17}, S_{22}$, and $S_{39}$ with the subscript numbers corresponding to the respective Ag layer thickness. On each sample we fabricate RMTs with four rotations and diameters of $d \sim 5 \mu \mathrm{m}$. Such RMTs are referred to as $\mathrm{RMT}_{6}, \mathrm{RMT}_{17}, \mathrm{RMT}_{22}$, and $\mathrm{RMT}_{39}$ where the subscript analogously denotes the thickness of the Ag layer rolled up in the microtubes. The resulting sample geometry is depicted in Fig. 1(a) showing a RMT along with the metal coated semiconductor multilayer structure from which the RMT was rolled up. The thickness of the semiconductor compound layer in the RMT is $30 \mathrm{~nm}$, while the thickness of the Ag layer varies in different RMTs $(6-39 \mathrm{~nm})$.

The spontaneous emission decay kinetics of the QWs were measured at low temperatures by time-resolved PL spectroscopy. We used a confocal micro-PL setup equipped with a continuous flow cryostat operating at liquid helium temperature $(4 \mathrm{~K})$. For timing resolution we used a timecorrelated single-photon counting (TCSPC) system [26].
This system is based on a picosecond pulsed diode laser emitting at $1.95 \mathrm{eV}$ as well as a single-photon sensitive avalanche photodiode. The decay kinetics is acquired at the PL emission peak $(1.64 \mathrm{eV})$ and lifetimes $\tau=1 / \Gamma$, with $\Gamma$ being the decay rate, are extracted by fitting a monoexponential decay to the decay kinetics. For resolving lifetimes below $80 \mathrm{ps}$, we used a streak camera system combined with a tunable Ti:sapphire laser with a pulse width of $600 \mathrm{fs}$ emitting at $1.72 \mathrm{eV}$. As sketched in Fig. 1(a), lifetimes $\tau$ of the QW are measured separately in the RMT $\left(\tau_{\mathrm{RMT}}\right)$ and, as a reference, also on the $\mathrm{Ag}$ coated $\left(\tau_{C}\right)$ flat substrate, from which the RMTs were rolled up, and on the flat substrate without metal coating $\left(\tau_{\mathrm{UC}}\right)$. The TCSPC system is used to measure the lifetimes $\tau_{C}$ and $\tau_{\mathrm{UC}}$. The lifetimes $\tau_{\mathrm{RMT}}$ are determined with the streak camera system, since these lifetimes are considerably below 80 ps. Transfer matrix calculations show that the Ag layers in the rolled-up multilayer structures reflect the excitation laser and absorb the subsequent PL emission of the excited QWs and thus significantly attenuate the signal from low-lying QWs. As the top QW layer [Fig. 1(c)] is unaffected by this attenuation we expect to predominantly observe the signal of the top QW layer in our far field experiment. As sketched in Fig. 1(d) we accordingly assume the RMT as a system consisting of a QW layer on top of a multilayer system composed of alternating metal and semiconductor layers.

We measured the lifetime of the QWs on $S_{6}, S_{17}, S_{22}$, and $S_{39}$ separately on the RMTs $\left(\tau_{\mathrm{RMT}}\right)$ and as a reference also in the flat metal coated $\left(\tau_{C}\right)$ and uncoated $\left(\tau_{\mathrm{UC}}\right)$ configuration [cf., Fig. 1(a)]. The results for $\tau_{\mathrm{RMT}}, \tau_{C}$, and $\tau_{\mathrm{UC}}$ are shown in Fig. 2(a). Figure 2(b) shows the lifetime of the QW in the microtubes $\tau_{\mathrm{RMT}}$ normalized with respect to the reference data $\tau_{C}$. The normalization $\left(\tau_{\mathrm{RMT}} / \tau_{C}\right)$ was 

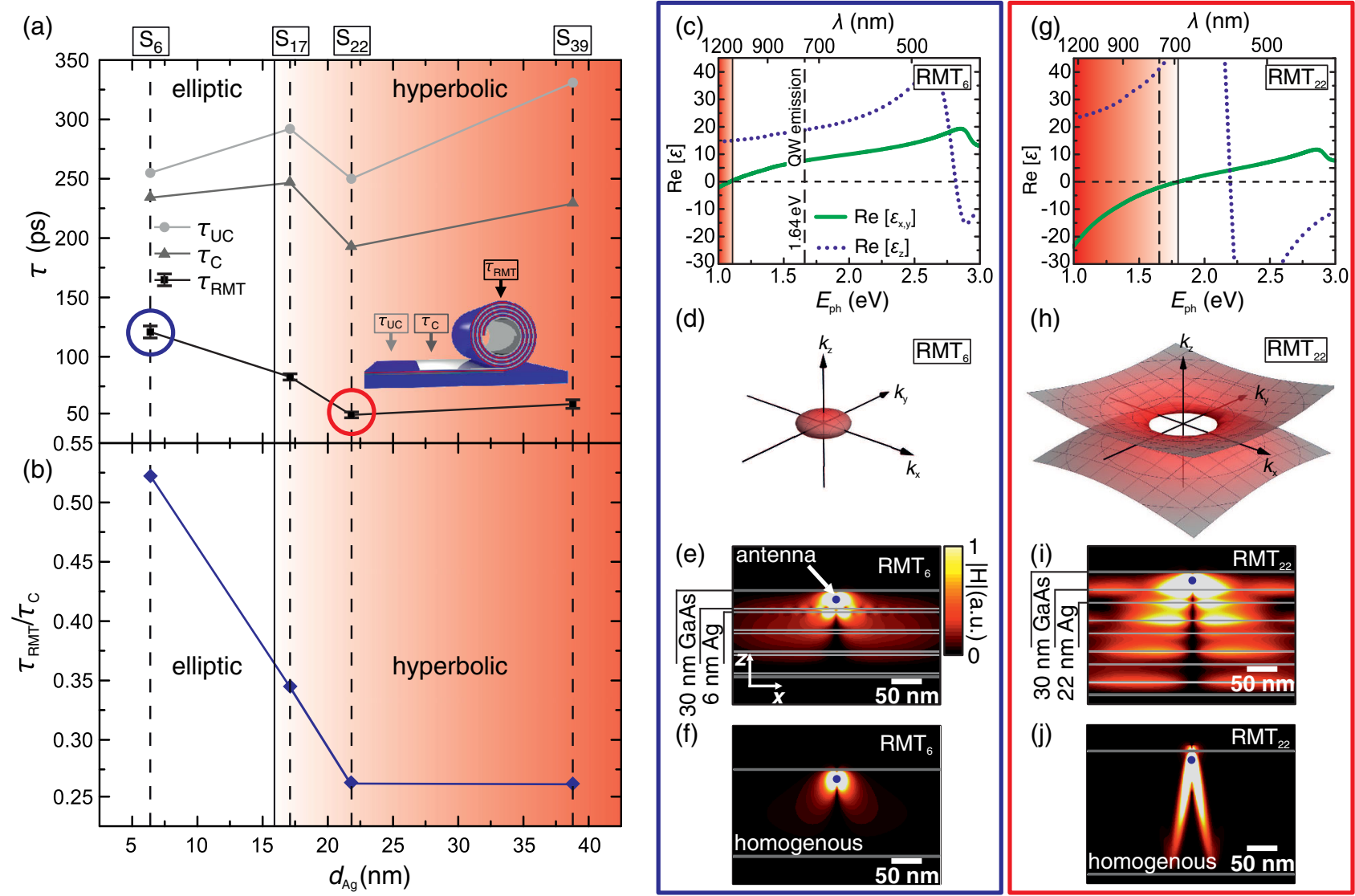

FIG. 2. (a) QW lifetimes $\tau_{\mathrm{UC}}$ (gray dots), $\tau_{C}$ (gray triangles), and $\tau_{\mathrm{RMT}}$ (black squares) on the samples $S_{6}, S_{17}, S_{22}$, and $S_{39}$. The top of the plot shows the corresponding sample label; the bottom shows the Ag layer thickness $d_{\mathrm{Ag}}$ on the respective samples. Red highlighted areas indicate Ag layer thicknesses $d_{\mathrm{Ag}}$ for which the RMTs exhibit a hyperbolic dispersion. (b) Normalized lifetime $\left(\tau_{\mathrm{RMT}} / \tau_{C}\right)$ plotted against the Ag layer thickness $d_{\mathrm{Ag}}$ on the respective samples. The blue framed figures (c)-(f) correspond to $\mathrm{RMT}_{6}\left(d_{\mathrm{Ag}}=6 \mathrm{~nm}\right)$ and the red framed figures $(\mathrm{g})-(\mathrm{j})$ to $\mathrm{RMT}_{22}\left(d_{\mathrm{Ag}}=22 \mathrm{~nm}\right)$. The QW lifetimes of RMT 6 and $\mathrm{RMT}_{22}$ are highlighted in (a) with a blue and red circle, respectively. [(c) and (g)] Real parts of the effective permittivities $\varepsilon_{x, y}$ and $\varepsilon_{z}$ for $\mathrm{RMT}_{6}$ and $\mathrm{RMT}_{22}$ based on an effective medium model. The red highlighted areas mark photon energies $E_{\mathrm{ph}}$ for which $\operatorname{Re}\left(\varepsilon_{x, y}\right)<0$ and $\operatorname{Re}\left(\varepsilon_{z}\right)>0$; the black dashed line indicates the QW's emission energy $E_{\mathrm{ph}, \mathrm{QW}}=1.64 \mathrm{eV}$. [(d) and (h)] Isofrequency plots of the dispersion relation for (d) RMT 6 and (h) RMT 22 for a photon energy of $E_{\mathrm{ph}, \mathrm{QW}}=1.64 \mathrm{eV}$. [(e) and (i)] Absolute values of the magnetic field amplitude in linear scale obtained from finitedifference time-domain (FDTD) simulations for a dipole antenna of length $6 \mathrm{~nm}$ (blue dot) emitting radiation at an energy $E=1.64 \mathrm{eV}$ in the top layer of (e) $\mathrm{RMT}_{6}$ and (i) $\mathrm{RMT}_{22}$. [(f) and (j)] Absolute values of the magnetic field amplitude for an idealized homogenous structure (i.e., no individual layers) with anisotropic material parameters $\varepsilon_{x, y}$ and $\varepsilon_{z}$ as determined from the effective medium model for (f) $\mathrm{RMT}_{6}$ and (j) $\mathrm{RMT}_{22}$

carried out in order to account for quenching effects of the metal compound in the microtubes and intrinsic lifetime variation of the utilized QW structures that are reflected in the reference data for $\tau_{C}$. Such intrinsic lifetime variations are caused by lateral allocated thickness and alloy fluctuations of the AlGaAs/GaAs/AlGaAs heterostructure during MBE growth that affect the local band structure of the quantum system and therefore alter the decay kinetics and the intrinsic spontaneous emission lifetimes [27,28]. The quenching effect can be observed when comparing the reference data $\tau_{C}$ and $\tau_{\mathrm{UC}}$. The lifetimes on metal coated areas $\tau_{C}$ are reduced compared to uncoated areas $\tau_{\mathrm{UC}}$ as a consequence of the metal layer in close proximity to the QW. The oscillating dipole fields of the QW can penetrate through the spacing layer $(10 \mathrm{~nm})$ and couple to surface plasmon polariton modes $[29,30]$ or lossy wave excitations $[30,31]$ at the metal interface in a near field coupling process. This offers additional decay channels for the QW that reduce its spontaneous emission lifetime.

We note that the normalized lifetime of the QW in the microtubes $\left(\tau_{\mathrm{RMT}} / \tau_{C}\right)$ [Fig. 2(b)] shows a reduction as $d_{\mathrm{Ag}}$ is increased; e.g., a comparison between $\mathrm{RMT}_{6}$ and $\mathrm{RMT}_{22}$ shows a reduction of $\left(\tau_{\mathrm{RMT}} / \tau_{C}\right)$ by a factor of 2 . The reduction of $\left(\tau_{\mathrm{RMT}} / \tau_{C}\right)$ can be well explained by the crossover from elliptic to hyperbolic dispersion between the different microtubes. In a first approach, we model the optical properties of the microtubes with an effective medium model. As can be seen in Figs. 1(b) and 1(c) 
the walls of the microtubes represent three-dimensional structures consisting of alternating layers of metal and dielectric. For such structures, the effective medium model yields an effective permittivity tensor $\varepsilon=\operatorname{diag}\left(\varepsilon_{x, y}, \varepsilon_{x, y}, \varepsilon_{z}\right)$ with uniaxial anisotropic components in plane $\varepsilon_{x, y}$ and out of plane $\varepsilon_{z}$ [32],

$$
\begin{gathered}
\epsilon_{x, y}=\frac{\eta \epsilon_{\mathrm{metal}}+\epsilon_{\text {dielectric }}}{1+\eta} \\
\frac{1}{\epsilon_{z}}=\frac{1}{1+\eta}\left(\frac{\eta}{\epsilon_{\text {metal }}}+\frac{1}{\epsilon_{\text {dielectric }}}\right) .
\end{gathered}
$$

The permittivity components $\varepsilon_{x, y}, \varepsilon_{z}$ both depend on the permittivities of the constituent materials $\left(\epsilon_{\text {metal }}\right.$ and $\left.\epsilon_{\text {dielectric }}\right)$ and on the layer thickness ratio between the metal and dielectric layer $\eta=d_{\text {metal }} / d_{\text {dielectric. }}$ We remark that changing the thickness ratio $\eta$ enables control over $\varepsilon_{x, y}$ and $\varepsilon_{z}$ over a wide range. Figures 2(c) and 2(g) show the real part of $\varepsilon_{x, y}$ (green solid) and $\varepsilon_{z}$ (blue dotted) for $\mathrm{RMT}_{6}$ $(\eta=0.22)$ and $\operatorname{RMT}_{22}(\eta=0.73)$, respectively, plotted against the photon energy $E_{\mathrm{ph}}$ where the $\mathrm{Ag}$ layer is the metal compound and the GaAs quantum structure is the dielectric compound. Values for complex permittivities of GaAs and Ag are taken from the data of Palik [33]. Red highlighted areas indicate photon energies $E_{\mathrm{ph}}$ for which $\operatorname{Re}\left(\varepsilon_{x, y}\right)<0$ and $\operatorname{Re}\left(\varepsilon_{z}\right)>0$; the black dashed line corresponds to the QW's emission energy $E_{\mathrm{ph}, \mathrm{QW}}=1.64 \mathrm{eV}$. With the dispersion relation for extraordinary (TMpolarized) waves in uniaxial anisotropic systems given by

$$
\frac{k_{x}^{2}+k_{y}^{2}}{\varepsilon_{z}}+\frac{k_{z}^{2}}{\varepsilon_{x, y}}=\frac{\omega^{2}}{c^{2}}
$$

we can evaluate the topology of the isofrequency surfaces of the RMTs on the respective samples. For $\mathrm{RMT}_{6}$, the isofrequency plot of the dispersion relation yields a closed ellipsoid in $k$ space as a consequence of $\operatorname{Re}\left(\varepsilon_{x, y}\right), \operatorname{Re}\left(\varepsilon_{z}\right)>0$ and $\operatorname{Re}\left(\varepsilon_{x, y}\right) \neq \operatorname{Re}\left(\varepsilon_{z}\right)$ at $E_{\mathrm{ph}, \mathrm{QW}}=1.64 \mathrm{eV}$ [Fig. 2(d)]. In contrast, for $\operatorname{RMT}_{22}$ we find $\operatorname{Re} \varepsilon_{x, y}<0$ and $\operatorname{Re}\left(\varepsilon_{z}\right)>0$ at $E_{\mathrm{ph}, \mathrm{QW}}=1.64 \mathrm{eV}$, which results in an isofrequency surface of hyperboloidal topology [Fig. 2(h)]. Even though for $\mathrm{RMT}_{22}$, Eq. (3) does not have a solution for small $k$ vectors, as can be seen from the hole in the center of the isofrequency surface, high- $k$ vectors are supported as the hyperbolic isofrequency surface is an open surface in $k$ space extending infinitely leading to a diverging PDOS.

In the effective medium model the transition from elliptic to hyperbolic dispersion occurs for samples with $d_{\mathrm{Ag}}>16 \mathrm{~nm}$, which agrees well with our data. In fact, $\mathrm{RMT}_{6}$ is expected to have elliptic dispersion and the QW in $\mathrm{RMT}_{6}$ has a relatively large lifetime of $\left(\tau_{\mathrm{RMT}} / \tau_{C}\right)=0.52$. By contrast, the lifetime of $\mathrm{RMT}_{22}\left[\left(\tau_{\mathrm{RMT}} / \tau_{C}\right)=0.25\right]$, which is expected to show hyperbolic dispersion, is significantly reduced. We attribute this difference in lifetime to the topological transition of the isofrequency surface to an open hyperboloidal surface giving rise to a diverging PDOS that induces a faster spontaneous emission of the embedded quantum emitter.

In $\operatorname{RMT}_{17}\left[\left(\tau_{\mathrm{RMT}} / \tau_{C}\right)=0.34\right]$ the lifetime is only moderately decreased compared to $\mathrm{RMT}_{6}\left[\left(\tau_{\mathrm{RMT}} / \tau_{C}\right)=\right.$ 0.52]. We assume that $\mathrm{RMT}_{17}$ does not yet show a distinct hyperbolic dispersion with a diverging PDOS since it lies in the crossover regime to the hyperbolic dispersion that is expected to be smeared out due to losses of the metallic components in the system [15]. Therefore, the reduction of the lifetime is less significant compared to $\mathrm{RMT}_{22}$, which agrees well with our expectation. $\mathrm{RMT}_{39}\left[\left(\tau_{\mathrm{RMT}} / \tau_{C}\right)=\right.$ $0.25]$ on the other hand is far in the hyperbolic regime and the lifetime is not reduced any further as compared to $\operatorname{RMT}_{22}\left[\left(\tau_{\mathrm{RMT}} / \tau_{C}\right)=0.25\right]$, which already shows distinct hyperbolic dispersion. Even though we effectively probe the lifetime of the top QW layer, since this layer provides the dominant contribution to the signal that we measure in our far field experiment, it is reasonable to assume that the lifetime of all quantum structures in the metamaterials is decreased in the hyperbolic dispersion regime. Radiation from lower lying QW layers could be coupled out to free space, in a subsequent step, by nanopatterning the structure, which has successfully been employed to couple out hyperbolic modes from a multilayer system [11].

FDTD simulations using the CST MICROWAVE STUDIO package very well support our experimentally observed results. We numerically determine the emission enhancement and analyze the spatial electromagnetic field distribution in $\mathrm{RMT}_{6}$ and $\mathrm{RMT}_{22}$. For this purpose we place a subwavelength dipole emitter of length $6 \mathrm{~nm}$ oriented in $z$-direction in the middle of the top layer of the microtubes [cf. Fig. 2(e)]. We use three-dimensional simulations with open boundary conditions (perfectly matched layers) and consider losses of the metallic components. The emission enhancement was simulated using the induced impedance model [34]. In this model, the emitter is a metal antenna and the effect of the environment on its radiation can be measured in terms of the antenna radiation resistance, which can be computed in CST MICROWAVE STUDIO. We remark that this classical antenna model is well suited to also describe the spontaneous emission enhancement of quantum emitters [34]. Care was taken in order to exclude the effect of mesh on the emitted power by providing a sufficiently dense mesh around the dipole, which is kept identical in all simulations. Computing the induced impedance for the light source embedded in $\mathrm{RMT}_{6}$ and $\mathrm{RMT}_{22}$, we determine an enhancement of the emitted power (i.e., a lifetime decrease) in $\mathrm{RMT}_{22}$ by a factor of 4.2 compared to $\mathrm{RMT}_{6}$. This result is the same order of magnitude as our experimental result (factor of 2). The electromagnetic field distribution in $\mathrm{RMT}_{22}$ reveals characteristic features of hyperbolic dispersion that are responsible for the emission 
enhancement. Figures 2(e) and 2(i) show the spatial distribution of the magnetic field amplitude in $\mathrm{RMT}_{6}$ and $\mathrm{RMT}_{22}$, respectively. We plot the magnetic field amplitude as it is free from unsteady boundary conditions at the layer interfaces. In Figs. 2(f) and 2(j) we show the simulation of an idealized structure with anisotropy parameters as determined from the effective medium model for $\mathrm{RMT}_{6}$, and $\mathrm{RMT}_{22}$, but simulated as homogenized media (i.e., without discrete layers). We note that in the idealized simulation for $\mathrm{RMT}_{22}$ [Fig. 2(j)] the radiation of the source is emitted in two preferred directions. Such directed wave beam propagation is characteristic for hyperbolic dispersion of optical waves [35,36], but also occurs for other wave phenomena such as spin waves [37] and acoustic waves [38] in media with engineered anisotropy. The directed beams are the consequence of high- $k$ vector modes that are expected to travel with a group velocity pointing in the same direction. For $\mathrm{RMT}_{6}$, the two distinctive beams do not occur since the sample features elliptic dispersion. We note that the layered samples show a similar behavior compared to the idealized structures. For $\mathrm{RMT}_{6}$, it can be additionally seen that fields are localized at the top metal layer, which is due to the excitation of a plasmonic mode at the metal-semiconductor interface in the vicinity of the emitter. For $\mathrm{RMT}_{22}$, field localizations as a consequence of plasmonic excitations can also be noticed but are distributed over the whole structure. Comparison between the idealized homogeneous [Fig. 2(j)] and the realized layered [Fig. 2(i)] simulation for $\mathrm{RMT}_{22}$ shows that also in the layered structure light propagation takes place in the expected directions shown for the ideal homogeneous sample. This demonstrates that also the realized samples with layer thicknesses in the order of $30 \mathrm{~nm}$ support characteristic features of hyperbolic dispersion giving rise to the lifetime enhancement of the integrated quantum wells as observed in our experiments.

In conclusion, we report on enhanced spontaneous emission rates of GaAs quantum wells embedded in rolled-up metamaterials. The metamaterials were fabricated by rolling up strained metal-semiconductor layers with incorporated quantum structures resulting in active metamaterials composed of identical functional layers. Time-resolved PL measurements supported by FDTD simulations reveal a reduction of the spontaneous emission lifetime of the integrated quantum structures as the thickness of the incorporated $\mathrm{Ag}$ layer in the metamaterials $d_{\mathrm{Ag}}$ is increased. Besides a contribution of quenching by the metal compound we identify a lifetime decrease as a signature of the topological transition to hyperbolic dispersion in our metamaterials that is predicted by a simple effective medium model and well supported by our electromagnetic simulations. Our study shows that the spontaneous emission rate of high-quality quantum-well structures with a narrow spectrum can be tailored when integrated in a metal-dielectric metamaterial system in which the emitters are part of an optical environment with controllable optical properties. Our results are an important step towards novel integrated quantum light sources with tailored emission properties.

We gratefully acknowledge financial support from the Deutsche Forschungsgemeinschaft Grant No. ME 3600/1 and GrK 1286 and CST, Darmstadt, Germany for support with their MICROWAVE STUDIO software.

K. Marvin Schulz and Hoan Vu contributed equally to this work.

*marvin.schulz@tuhh.de

†hvu@physnet.uni-hamburg.de

[1] R. A. Shelby, D. R. Smith, and S. Schultz, Science 292, 77 (2001).

[2] J. Valentine, S. Zhang, T. Zentgraf, E. Ulin-Avila, D. A. Genov, G. Bartal, and X. Zhang, Nature (London) 455, 376 (2008).

[3] N. Liu, H. Guo, L. Fu, S. Kaiser, H. Schweizer, and H. Giessen, Nat. Mater. 7, 31 (2008).

[4] A. Rottler, M. Harland, M. Broell, S. Schwaiger, D. Stickler, A. Stemmann, C. Heyn, D. Heitmann, and S. Mendach, Appl. Phys. Lett. 100, 151104 (2012).

[5] Z. Liu, H. Lee, Y. Xiong, C. Sun, and X. Zhang, Science 315, 1686 (2007).

[6] S. Schwaiger, M. Broll, A. Krohn, A. Stemmann, C. Heyn, Y. Stark, D. Stickler, D. Heitmann, and S. Mendach, Phys. Rev. Lett. 102, 163903 (2009).

[7] J. B. Pendry, D. Schurig, and D. R. Smith, Science 312, 1780 (2006).

[8] A. Rottler, B. Kruger, D. Heitmann, D. Pfannkuche, and S. Mendach, Phys. Rev. B 86, 245120 (2012).

[9] H. N. S. Krishnamoorthy, Z. Jacob, E. Narimanov, I. Kretzschmar, and V. M. Menon, Science 336, 205 (2012).

[10] T. Tumkur, G. Zhu, P. Black, Y. A. Barnakov, C. E. Bonner, and M. A. Noginov, Appl. Phys. Lett. 99, 151115 (2011).

[11] D. Lu, J. J. Kan, E. E. Fullerton, and Z. Liu, Nat. Nanotechnol. 9, 48 (2014).

[12] T. Galfsky, H. N. S. Krishnamoorthy, W. Newman, E. E. Narimanov, Z. Jacob, and V. M. Menon, Optica 2, 62 (2015).

[13] L. Ferrari, D. Lu, D. Lepage, and Z. Liu, Opt. Express 22, 4301 (2014).

[14] Y. Zhang, D. Han, D. Du, G. Huang, T. Qiu, and Y. Mei, Plasmonics 10, 949 (2015).

[15] Z. Jacob, J.-Y. Kim, G. Naik, A. Boltasseva, E. Narimanov, and V. Shalaev, Appl. Phys. B 100, 215 (2010).

[16] C. L. Cortes, W. Newman, S. Molesky, and Z. Jacob, J. Opt. 14, 063001 (2012).

[17] A. Poddubny, I. Iorsh, P. Belov, and Y. Kivshar, Nat. Photonics 7, 948 (2013).

[18] V. P. Drachev, V. A. Podolskiy, and A. V. Kildishev, Opt. Express 21, 15048 (2013).

[19] V. Y. Prinz, V. A. Seleznev, A. K. Gutakovsky, A. V. Chehovskiy, V. V. Preobrazhenskii, M. A. Putyato, and T. A. Gavrilova, Physica E (Amsterdam) 6, 828 (2000). 
[20] O. G. Schmidt and K. Eberl, Nature (London) 410, 168 (2001).

[21] C. Strelow, H. Rehberg, C. M. Schultz, H. Welsch, C. Heyn, D. Heitmann, and T. Kipp, Phys. Rev. Lett. 101, 127403 (2008).

[22] F. Balhorn, C. Bausch, S. Jeni, W. Hansen, D. Heitmann, and S. Mendach, Phys. Rev. B 88, 054402 (2013).

[23] A. Rottler, M. Harland, M. Bröll, M. Klingbeil, J. Ehlermann, and S. Mendach, Phys. Rev. Lett. 111, 253901 (2013).

[24] J. Zhang, J. Li, S. Tang, Y. Fang, J. Wang, G. Huang, R. Liu, L. Zheng, X. Cui, and Y. Mei, Sci. Rep. 5, 15012 (2015).

[25] S. Tang, Y. Fang, Z. Liu, L. Zhou, and Y. Mei, Lab Chip 16, 182 (2016).

[26] W. Becker, A. Bergmann, M. A. Hink, K. Konig, K. Benndorf, and C. Biskup, Microsc. Res. Tech. 63, 58 (2004).

[27] K. L. Campman, H. Schmidt, A. Imamoglu, and A. C. Gossard, Appl. Phys. Lett. 69, 2554 (1996).

[28] T. Unuma, M. Yoshita, T. Noda, H. Sakaki, and H. Akiyama, J. Appl. Phys. 93, 1586 (2003).

[29] W. H. Weber and C. F. Eagen, Opt. Lett. 4, 236 (1979).
[30] I. Pockrand, A. Brillante, and D. Mobius, Chem. Phys. Lett. 69, 499 (1980).

[31] G. W. Ford and W. H. Weber, Phys. Rep. 113, 195 (1984).

[32] B. Wood, J. B. Pendry, and D. P. Tsai, Phys. Rev. B 74, 115116 (2006).

[33] E. D. Palik, Handbook of Optical Constants of Solids (Academic, New York, 1985).

[34] A. E. Krasnok, A. P. Slobozhanyuk, C. R. Simovski, S. A. Tretyakov, A. N. Poddubny, A. E. Miroshnichenko, Y.S. Kivshar, and P. A. Belov, Sci. Rep. 5, 12956 (2015).

[35] Z. Jacob, L. V. Alekseyev, and E. Narimanov, Opt. Express 14, 8247 (2006).

[36] S. Schwaiger, A. Rottler, M. Broll, J. Ehlermann, A. Stemmann, D. Stickler, C. Heyn, D. Heitmann, and S. Mendach, Phys. Rev. B 85, 235309 (2012).

[37] S. Mansfeld, J. Topp, K. Martens, J. N. Toedt, W. Hansen, D. Heitmann, and S. Mendach, Phys. Rev. Lett. 108, 047204 (2012).

[38] C. Shen, Y. Xie, N. Sui, W. Wang, S. A. Cummer, and Y. Jing, Phys. Rev. Lett. 115, 254301 (2015). 